\title{
Logic Programming and Nonmonotonic Reasoning: From Theory to Systems and Applications
}

\author{
Nicola Leone \\ Department of Mathematics, University of Calabria, 87036 Rende (CS), Italy \\ leoneamat.unical.it
}

\begin{abstract}
LPNMR is based on very solid theoretical foundations. After nearly twenty years of research, LPNMR languages are expressively rich, and their semantic and computational properties are well understood today. Moreover, in the last decade the LPNMR community has focused also on systems implementation, and, after the pioneering work carried out in DLV and Smodels, many efficient LPNMR systems are now available.

The main challenge of LPNMR now concerns applications. Two key questions are:

- Is LPNMR technology mature enough for the development of industrial applications?

- Can LPNMR be attractive also for the market?

In this talk, we will address the above questions. We will report also some feedback that we have got "from the field", in the collaboration with a Calabrian spin-off company, which is developing three Knowledge Management products based on the DLV system, in a joint venture with a US company. We will present our vision on the future development of LPNMR, pointing out promising application areas where LPNMR can be profitably exploited, and specifying the key theoretical and practical issues to be addressed in order to enhance the applicability of LPNMR.
\end{abstract}

